\title{
3 Research Soure \\ Development of an Unpowered Energy Recycling Exoskeleton for Walking Assist
}

\section{kun wang}

Tianjin institute of environmental and operational medicine https://orcid.org/0000-0002-2106-7808

\section{Xiujie Gao}

Tianjing institute of environmental and operational medicine

\section{Kefeng Ma}

Tianjin institute of environmental and operational medicine

\section{Xiaojun She}

Tianjin institute of environmental and operational medicine

\section{Honglian Yang}

Tianjin institute of environmental and operational medicine

Bo Cui ( $\boldsymbol{\nabla}$ iamcuib@sina.com)

Tianjin institute of environmental and operational medicine

\section{Research}

Keywords: Walking assist, Energy recycling, Unpoweredexoskeleton, EMG

Posted Date: July 17th, 2020

DOI: https://doi.org/10.21203/rs.3.rs-42279/v1

License: (c) (i) This work is licensed under a Creative Commons Attribution 4.0 International License. Read Full License 


\section{Abstract \\ Background}

A reduction of energy used during walking could present a significant advantage for both healthy individuals and those with reduced mobility. Current unpowered exoskeletons have shown a net reduction in energy consumption for walking, but commonly with a clutch and assist on the heel. This paper presents the development of a lightweight energy recycling exoskeleton without a clutch and assists on the forefoot.

\section{Methods}

Eight healthy participants were tested during walking at $1.25 \mathrm{~m} / \mathrm{s}$ on a treadmill wearing exoskeletons of four kinds of conditions. Electromyography $(E M G)$ of the soleus muscle and gastrocnemius muscle were collected.

\section{Results}

Our results showed that the novel exoskeleton could make the peak and average EMG-values of soleus muscles drop about $13 \%$ and $8 \%$ respectively, and that of gastrocnemius muscles drop about $12 \%$ and $13 \%$ respectively.

\section{Conclusions}

The results of the present work demonstrate for the first time that the novel energy recycling exoskeleton can improve the walking economy, and this device could be feasibly worn for a broad range of individuals.

\section{Introduction}

Human walking has evolved over a long period of time for locomotion which minimizes its energy cost while the amount of energy spent on walking is still larger than any other activity in daily life[1]. The improvement of the walking economy could thus be beneficial for both healthy individuals and those with reduced mobility[2]. Many attempts have been made to assist walking especially unpowered assistive devices have demonstrated the capacity to capture and release energy throughout the gait cycle, and have shown the ability to achieve a net reduction in energy consumption $[3,4]$.

Theoretically, the network requirement for steady-state walking is zero[5]. Dissipation occurs in muscles or other soft tissues in the body, with a major loss occurring during heel strike collisions of walking[5, 6]. Total work may be minimized if the energy losses could be reduced[7]. Steven H. Collins' research 
successfully recycled the collision energy using an artificial foot and confirmed a net metabolic energy expenditure reduction[6]. However, the timing and the amount of energy insertion into the gait cycle is still an unresolved challenge for unpowered exoskeleton[7-11]. In humans, the power for walk is mainly provided by the positive work of the ankle and hip[12]. The greatest portion of energy waste occurs in muscles such as soleus muscle and gastrocnemius muscle during the push-off phase of the walking cycle $[4,13,14]$.

In practice, the assist devices should be lightweight to ensure a net positive energy reduction of walk. What's more, the human body needs to adapt to additional assistance[15], so it may be more comfortable to achieve walking energy consumption reduction without feeling the additional assist force. For the above purpose, we developed and tested an unpowered wearable energy recycling exoskeleton to improve the economy of human locomotion. Different from previous studies[16-18], the exoskeleton utilizes a very simple torsion spring structure to capture collision energy at the heel and returns it at the forefoot for push-off which is more in line with the foot force habit of human walking compared to returning energy at the heel.

\section{Methods}

\section{Exoskeleton Design}

A double torsion spring structure was used to capture the energy of heel strike collisions and recycles it as positive ankle work. As shown in Fig. 1, one end of the torsion spring arm is connected and serves as the fixed arm on the ground support. The other end of the torsion spring arm serves as the force application arm with 90-degree bend inward at the end which inserts and fixed to the back heel sole. Two metal bars in the middle support and fix on the sole of the forefoot. Thus the exoskeleton is fixed on the sole by the end of the force application arm and the middle metal bar.

The Exoskeleton was fixed to the sole by drilling holes in the sole, so the position of the holes determines the assisting site of an exoskeleton. The larger the torsion spring angle is, the more collision energy can be obtained. But a large torsion spring angle would make the exoskeleton wear like high heels. So the torsion spring angle is set as 15 degree when all feet on the ground the torsion spring angle is compressed to about 0 degrees. Through the above design, the exoskeleton does not affect the posture of the foot during the standing phase and push-off phase.

The smaller the torsion spring stiffness, the smaller the energy loss of the collision between the torsion spring arm and the ground. As the compression of the torsion spring angle is fixed, and in order to capture as much energy as possible from the heel landing, the torsion spring stiffness shall be designed so that the sole of the back heel just touches the ground when walking. On the other hand, large torsion spring stiffness would reduce comfort and even change the gait. We designed three kinds of torsion spring stiffnesses which make the support force of the metal bar about equal to, larger than and less than the bodyweight respectively when the back heel touches the ground. 


\section{Energy recycling mechanism}

As shown in Fig. 2, the end of the force application arm is pressed down to capture the collision energy when the heel lands, then during the standing phase, the plantar pressure gradually changes from the back to the front, so that the force on the force application arm changes from the back sole to the middle metal bar. During the push-off phase the torsion spring force application arm releases energy in the middle metal bar. We didn't design a clutch to control the storage and release of energy, alternatively, we simply set the boost site near the forefoot to achieve energy release during the push-off phase. And the energy release time could be adjusted through changing the position of the boost point.

\section{Prototype fabrication}

The material of the exoskeleton is SUS304-WPB, and the number of torsion spring turns is 1 with an average spiral diameter of $13 \mathrm{~mm}$, and the arm length of torsion spring is $150 \mathrm{~mm}$. The distance between the metal bar and the front end of the torsion spring is $60 \mathrm{~mm}$. In order to compare the assistant effect of the torsion spring with different stiffnesses, three kinds of exoskeletons were made according to the different diameter of torsion spring. The parameters of the exoskeletons were shown in Table 1. Due to the poor effect of assistant effect when the torsion spring angle is 15 degrees, we set the torsion spring angle to 30 degrees, and then compress the angle between the torsion springs to 15 degrees with the cable tie. A kind of casual shoes with holes in the sole was used to install the exoskeleton as shown in Fig. 3.

Table 1

The parameters of different kinds of exoskeletons.

\begin{tabular}{|lllll|}
\hline & $\begin{array}{l}\text { Wire diameter } \\
(\mathbf{m m})\end{array}$ & $\begin{array}{l}\text { Total weight } \\
(\mathbf{g})\end{array}$ & $\begin{array}{l}\text { Stiffness } \\
(\mathbf{k g} \cdot \mathbf{m m} / \mathrm{deg})\end{array}$ & $\begin{array}{l}\text { Support force of the metal } \\
\text { bar }(\mathrm{kg})\end{array}$ \\
\hline $\begin{array}{l}\text { Torsion } \\
\text { spring 1 }\end{array}$ & 4 & 44.7 & 31.8 & 31.8 \\
\hline $\begin{array}{l}\text { Torsion } \\
\text { spring 2 }\end{array}$ & 5 & 70.33 & 77.8 & 77.8 \\
\hline $\begin{array}{l}\text { Torsion } \\
\text { spring 3 }\end{array}$ & 6 & 101.98 & 161.2 & 161.2 \\
\hline
\end{tabular}

\section{Participants}

We tested eight healthy participants $(7 \bigotimes, 1 \bigotimes, 70 \pm 5 \mathrm{~kg}, 170 \pm 5 \mathrm{~cm}, 23 \pm 2$ years, values are mean \pm standard error) during walking at $1.25 \mathrm{~m} / \mathrm{s}$ on a treadmill. The walking speed of $1.25 \mathrm{~m} / \mathrm{s}$ was selected to reflect the preferred walking speed of healthy adults and to be similar to the speed that is used in most exoskeleton studies with healthy participants. All human subject studies were approved by the Academy of military medicine and the subjects gave informed written consent prior to participation. 


\section{Protocol}

We have prepared four pairs of the same shoes. Three pairs of shoes are equipped with the springs with different stiffnesses and a pair of the shoes serve as the control. Before the testing protocol, the participants were allowed 18 min of habituation to each pair of shoes. Then the subjects walked with each pair of shoes for two minutes during testing and rested $10 \mathrm{~min}$ while they changed the shoes. We collected electromyography (EMG, Biopac, $2000 \mathrm{~Hz}$ ) of the soleus muscle and gastrocnemius muscle. A common camera was used to record the lower limb movement to distinguish the gait cycle.

\section{Data Processing}

We rectified the EMG data, applied a bandpass filter $(50-450 \mathrm{~Hz})$, and then calculated a moving root mean square (RMS) with a window of $100 \mathrm{~ms}$ to reflect the instant activation of muscle similar to Philippe Malcolm's work. We normalized the EMG data to the average peak value per stride. To ensure steady-state had been reached, we averaged across the final 10 strides from each trial and then plotted comparisons between conditions similar to Matthew B. Yandell's work. Integral EMG (iEMG) of each stride was calculated to reflect the average muscle activation. Statistical analysis was conducted with Origin 8.5 , and the data were expressed as means \pm standard deviation. Paired $t$ test was used to verify the effect of the device on the EMG results across the stride.

\section{Results}

The soleus muscle EMG results of a typical subject were shown in Fig. 4. Over the stride, the peak EMGvalues of torsion spring $1(0.92 \pm 0.06)$ and the torsion spring $2(0.78 \pm 0.05)$ were significantly reduced compared to the control $(1.13 \pm 0.06)$, and we observed significant reductions in average soleus activity for the torsion spring $1(0.26 \pm 0.02)$ relative to the control $(0.27 \pm 0.02)$. Due to the high rigidity of the torsion spring 3 , it's hard for the lighter to step on and even changed the normal gait of the subjects. The soleus muscle EMG results of the torsion spring 3 were higher than the control.

For all the eight subjects, the peak EMG-values and average EMG-values of soleus muscle were calculated as shown in Fig. 5. The average EMG-values dropped about $8 \%$ for the torsion spring 1 ( $0.33 \pm$ 0.09 ) relative to the control $(0.36 \pm 0.08)$, and the peak EMG-values dropped about $13 \%$ and $11 \%$ for the torsion spring $1(0.86 \pm 0.11)$ and torsion spring $2(0.88 \pm 0.1)$ respectively relative to the control $(0.99 \pm$ $0.14)$.

The gastrocnemius muscle EMG results of the typical subject were shown in Fig. 6 . Similar to the soleus muscle results, the peak EMG-values of the torsion spring $1(0.85 \pm 0.1)$ and the torsion spring $2(0.95 \pm$ $0.06)$ were significantly lower than the control $(1.08 \pm 0.08)$, and the average soleus activity of the torsion spring $1(0.27 \pm 0.04)$ and the torsion spring $2(0.29 \pm 0.03)$ were also significantly lower than the control $(0.35 \pm 0.02)$. 
The peak EMG-values and average EMG-values of gastrocnemius muscle for all the subjects were shown in Fig. 7. The average EMG-values dropped about $13 \%$ and $5 \%$ for the torsion spring $1(0.25 \pm 0.02)$ and torsion spring $2(0.28 \pm 0.02)$ relative to the control $(0.29 \pm 0.04)$, and the peak EMG-values dropped about $12 \%$ and $16 \%$ for the torsion spring $1(0.92 \pm 0.11)$ and torsion spring $2(0.88 \pm 0.11)$ respectively relative to the control $(1.05 \pm 0.14)$.

\section{Discussion}

Here we present a novel design for an unpowered exoskeleton that captures the collision energy when the heel lands and releases energy on the forefoot. The EMG results demonstrated its good walking assistance effect. For the torsion 1, the peak and average EMG-values of soleus muscles dropped about $13 \%$ and $8 \%$ respectively, and that of gastrocnemius muscles dropped about $12 \%$ and $13 \%$ respectively compared to the results of control, so the walking assistance effect of this kind of exoskeleton is close to Matthew B. Yandells' work which has an average EMG-values reduction of $17 \%$ of soleus muscles[3]. We found that the relationship between the assist effect and spring stiffness is not simply linear, as shown in Fig. 4 and Fig. 5, the soleus muscle EMG results of torsion spring 1 were higher than that of torsion spring 2 while the soleus muscle EMG results were the opposite as shown in Fig. 6 and Fig. 7, and the torsion spring 3 EMG results were almost always higher than the control results because it influenced the normal gait of the subjects. Larger spring stiffness would influence the normal gait as the torsion spring 3 results show. Therefore, more tests were needed to find out the relationship between the assist effect and spring stiffness.

\section{Design Innovations}

The exoskeleton has the advantage of lightweight, low cost to manufacture, and very simple structure. This new design has the potential to be used on a wide scale to benefit a broad range of individuals throughout society including assisting the elderly or some patients with lower extremity injury and assisting users who walk for a long time to reduce fatigue, such as warehouse workers, hospital nurses, and soldiers. Our main innovative point is that we make the assist process more in line with the foot force habit of human walking. Most of the unpowered walking assist exoskeletons set the force assist point on the heel to provide torque for ankle joint in the push-off phase while the heel is not subjected to force in push-off phase during normal walking[1, 3, 7, 19]. So this design is easier for wearers to adapt to. To our knowledge, this is the first work to consider the force assist point on the plantar of energy recycling exoskeleton for the comfort of walking assist. What's more, our design is quite simple and lightweight, the torsion spring 1 is just $44.7 \mathrm{~g}$ per leg which is the lightest among the recent unpowered walking assist exoskeleton as shown in Table 2. 
Table 2

Mass of Recent Exoskeletons.

\begin{tabular}{|lllll|}
\hline & $\begin{array}{l}\text { Collins, } \\
\text { Wiggin } \\
\text { \& Sawicki } \\
\text { 2015[1] }\end{array}$ & $\begin{array}{l}\text { Mooney, } \\
\text { Rouse } \\
\text { \& Herr } \\
\text { 2014b[20] }\end{array}$ & $\begin{array}{l}\text { Marc Doumit\& } \\
\text { Leclair, J.[7] }\end{array}$ & $\begin{array}{l}\text { Karl E. Zelik \& Matthew B. } \\
\text { Yandell[3] }\end{array}$ \\
\hline $\begin{array}{l}\text { Total } \\
\text { Mass }\end{array}$ & 816-1006 g & $4660 \mathrm{~g}$ & $1350 \mathrm{~g}$ & $459 \mathrm{~g}$ \\
\hline
\end{tabular}

\section{Prototype Limitations}

The current prototype was developed, built, and tested to demonstrate one implementation of our design concept. The prototype would benefit from improvements in materials and manufacturing techniques to improve the longevity and robustness of the device. The weight of our current prototype could be reduced by changing the metal torsion spring arm into a lighter material. When the straight metal torsion spring arm land on the ground, it produces large noise and a counter-clockwise torque to the sole of the foot which needed extra muscle strength for offset. Turning the torsion spring arm into a certain bending shape and making the torsion spring arm surface soft may solve the above defects. Furthermore, the current prototype just captures collision energy at heel while the energy that can be captured by the standing phase of a gait cycle is ignored. So the assist effect could be further increased by design improvement.

\section{Experiment Limitations}

Due to the limitation of our experimental conditions, only EMG was monitored to evaluate the effect of assistance. Although EMG is an objective and direct indicator of the exoskeleton assist effect, more comprehensive experiments should be carried out such as motion capture, plantar pressure recording, and metabolic energy expenditure monitoring.

\section{Conclusion}

In summary, this work proposed a novel unpowered exoskeleton with the advantage of lightweight, low cost to manufacture, very simple structure and the assist process is more in line with the foot force habit of human walking. We replaced the clutch with controlling the energy release by changes in the plantar angle which simplified the overall structure of the device and considered providing assistance in the forefoot for the first time. The EMG results confirmed the beneficial effect for walking of this exoskeleton, and this device could be feasibly worn for a broad range of individuals.

\section{Declarations}

Ethics approval and consent to participate 
All human subject studies were approved by the Academy of military medicine and the subjects gaveinformed written consent prior to participation.

\section{Consent for publication}

Not applicable.

\section{Availability of data and materials}

All data generated or analysed during this study are included in this published article.

\section{Competing interests}

The authors declare that they have no competing interests.

\section{Funding}

This study was financially supported by Innovation Foundation of Academy of Military Medical Sciences.

\section{Authors' contributions}

The manuscript was written through contributions of all authors. All authors have given approval to the final version of the manuscript.\#These authors contributed equally.

\section{Acknowledgment}

Not applicable.

\section{References}

1. Collins SH, Wiggin MB, Sawicki GS. Reducing the energy cost of human walking using an unpowered exoskeleton [J]. Nature. 2015;522(7555):212-5.

2. Chen $B, Z i B$, Zeng Y, et al. Ankle-foot orthoses for rehabilitation and reducing metabolic cost of walking: Possibilities and challenges [J]. Mechatronics. 2018;53:241-50.

3. Yandell MB, Tacca JR, Zelik KE. Design of a Low Profile, Unpowered Ankle Exoskeleton That Fits Under Clothes: Overcoming Practical Barriers to Widespread Societal Adoption [J]. IEEE Trans Neural Syst Rehabil Eng. 2019;27(4):712-23.

4. Nuckols RW, Dick TJM, Beck ON, et al. Ultrasound imaging links soleus muscle neuromechanics and energetics during human walking with elastic ankle exoskeletons [J]. Sci Rep. 2020;10(1):3604-19.

5. Zelik KE, Huang TW, Adamczyk PG, et al. The role of series ankle elasticity in bipedal walking [J]. J Theor Biol. 2014;346:75-85.

6. Collins $\mathrm{SH}$, Kuo $A D$. Recycling energy to restore impaired ankle function during human walking [J]. PLoS One. 2010;5(2):e9307. 
7. Leclair J, Pardoel S, Helal A, et al. Development of an unpowered ankle exoskeleton for walking assist [J]. Disabil Rehabil Assist Technol, 2018: 1-13.

8. Malcolm P, Derave W, Galle S, et al. A simple exoskeleton that assists plantarflexion can reduce the metabolic cost of human walking [J]. PLoS One. 2013;8(2):e56137.

9. Malcolm P, Quesada RE, Caputo JM, et al. The influence of push-off timing in a robotic ankle-foot prosthesis on the energetics and mechanics of walking [J]. J Neuroeng Rehabil. 2015;12:21.

10. Galle S, Malcolm P, Collins SH, et al. Reducing the metabolic cost of walking with an ankle exoskeleton: interaction between actuation timing and power [J]. J Neuroeng Rehabil. 2017;14(1):35.

11. Quinlivan B, Lee S, Malcolm P, et al. Assistance magnitude versus metabolic cost reductions for a tethered multiarticular soft exosuit [J]. Science Robotics. 2017;2:eaah4416.

12. Ding Y, Panizzolo FA, Siviy C, et al. Effect of timing of hip extension assistance during loaded walking with a soft exosuit [J]. J Neuroeng Rehabil. 2016;13(1):87.

13. Sawicki GS, Khan NS. A Simple Model to Estimate Plantarflexor Muscle-Tendon Mechanics and Energetics During Walking With Elastic Ankle Exoskeletons [J]. IEEE Trans Biomed Eng. 2016;63(5):914-23.

14. Steele KM, Jackson RW, Shuman BR, et al. Muscle recruitment and coordination with an ankle exoskeleton [J]. J Biomech. 2017;59:50-8.

15. Malcolm P, Galle S, Derave W, et al. Bi-articular Knee-Ankle-Foot Exoskeleton Produces Higher Metabolic Cost Reduction than Weight-Matched Mono-articular Exoskeleton [J]. Front Neurosci. 2018;12:69.

16. Zhang J, Fiers P, Witte KA, et al. Human-in-the-loop optimization of exoskeleton assistance during walking [J]. 2017, 356(6344): 1280-4.

17. Dembia CL, Silder A, Uchida TK, et al. Simulating ideal assistive devices to reduce the metabolic cost of walking with heavy loads [J]. PLoS One. 2017;12(7):e0180320.

18. van Dijk W, Meijneke C, van der Kooij H. Evaluation of the Achilles Ankle Exoskeleton [J]. IEEE Trans Neural Syst Rehabil Eng. 2017;25(2):151-60.

19. Lee JD, Mooney LM, Rouse EJ. Design and Characterization of a Quasi-Passive Pneumatic FootAnkle Prosthesis [J]. IEEE Trans Neural Syst Rehabil Eng. 2017;25(7):823-31.

20. Mooney LM, Rouse EJ, Herr HM. Autonomous exoskeleton reduces metabolic cost of human walking during load carriage [J]. J Neuroeng Rehabil. 2014;11:80.

\section{Figures}



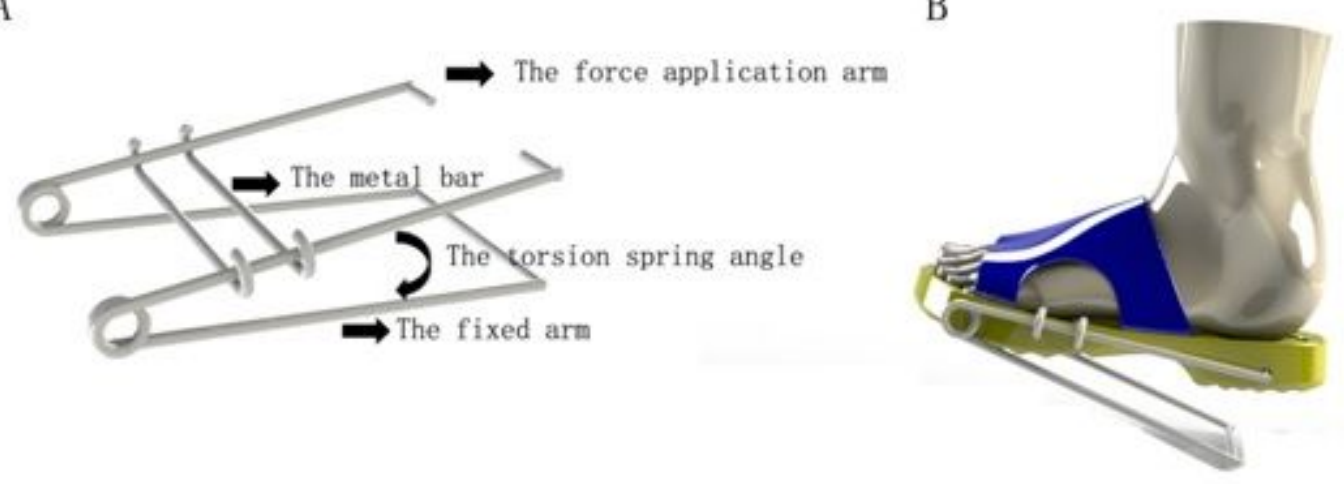

\section{Figure 1}

Overall design of the exoskeleton:A is the exoskeleton structure diagram, $B$ is an example of the exoskeleton assembly to the sole.

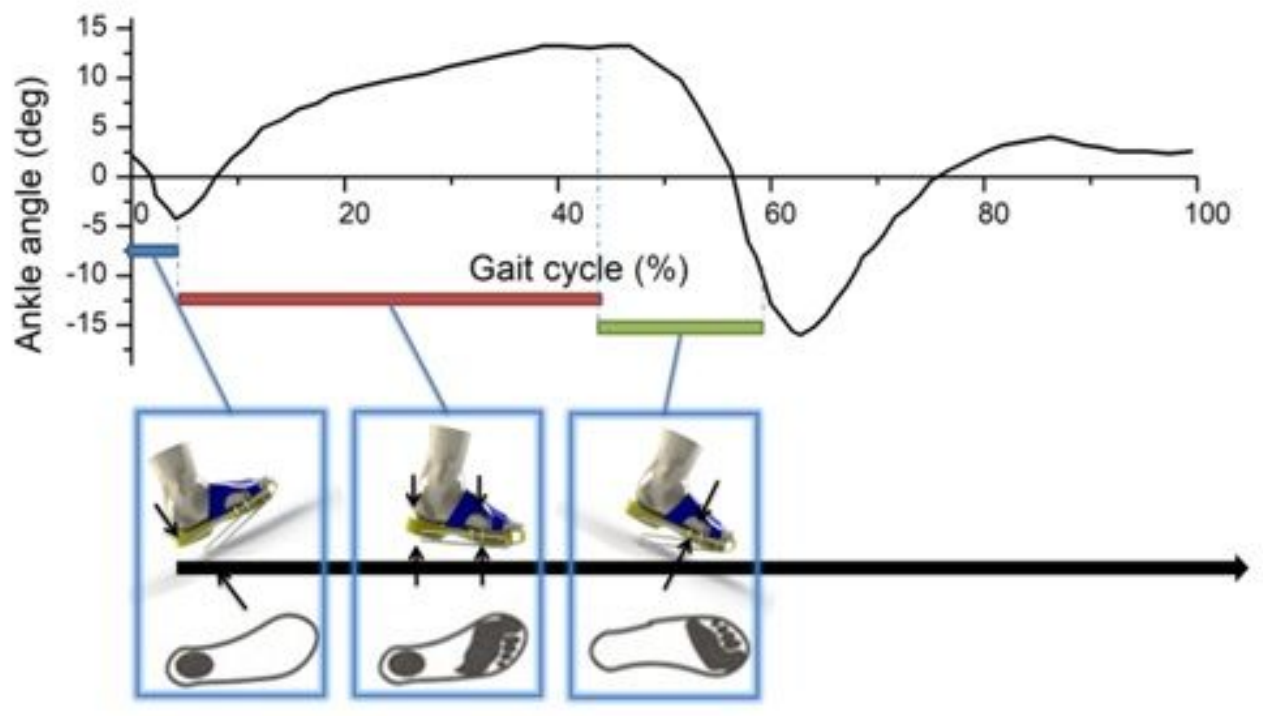

Figure 2

The exoskeleton assistance process during a gait cycle. 

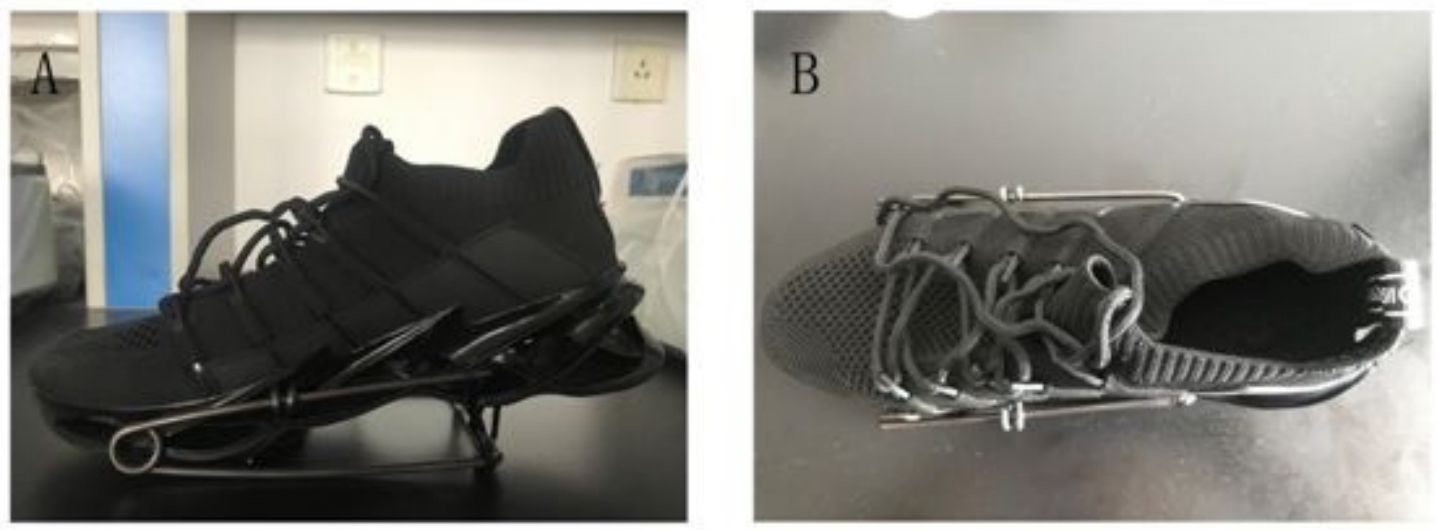

Figure 3

Photos of the exoskeleton prototype: A is the front view, B is the top view.
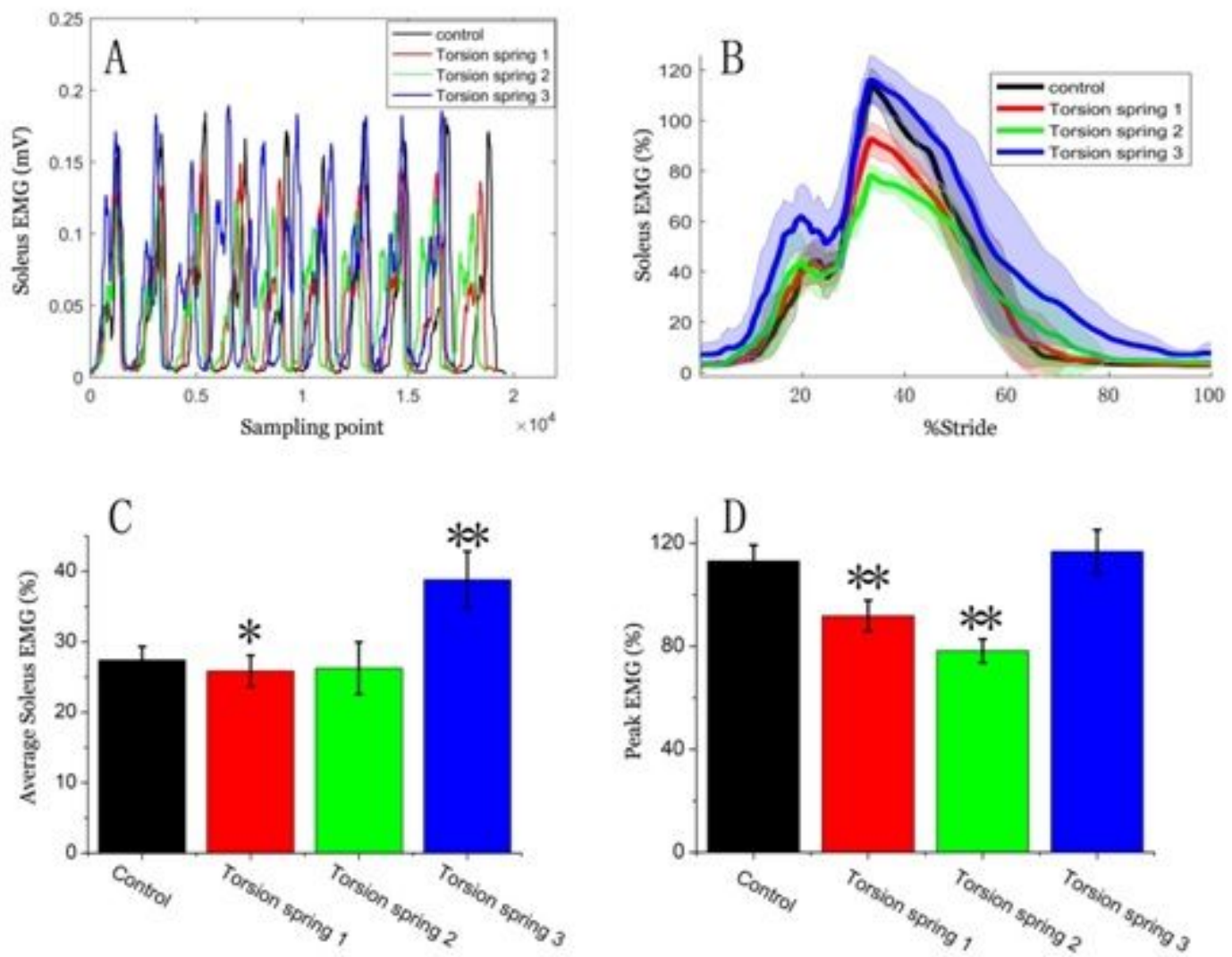

\section{Figure 4}

The soleus muscle EMG results of a typical subject.(A) RMS of the 10 strides.(B) Normalized RMS between conditions,colored lines indicate the population average of different conditions and Shaded areas indicate standard error. (C)iEMG of different conditions. (D) Peak EMG of different conditions.T test, $n=10,{ }^{*} \mathrm{p} \leq 0.05$. ** $\mathrm{p} \leq 0.01$. 

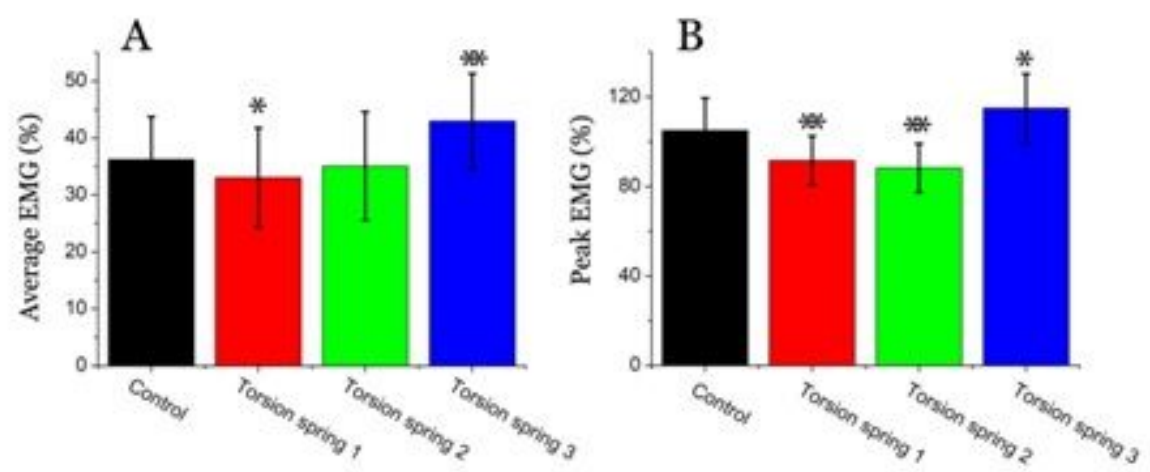

\section{Figure 5}

The soleus muscle EMG results of all subjects: $A$ is theiEMG result, $B$ is the Peak EMG result. T test, $n=80$, ${ }^{*} \mathrm{p} \leq 0.05 .{ }^{*} \mathrm{p} \leq 0.01$.
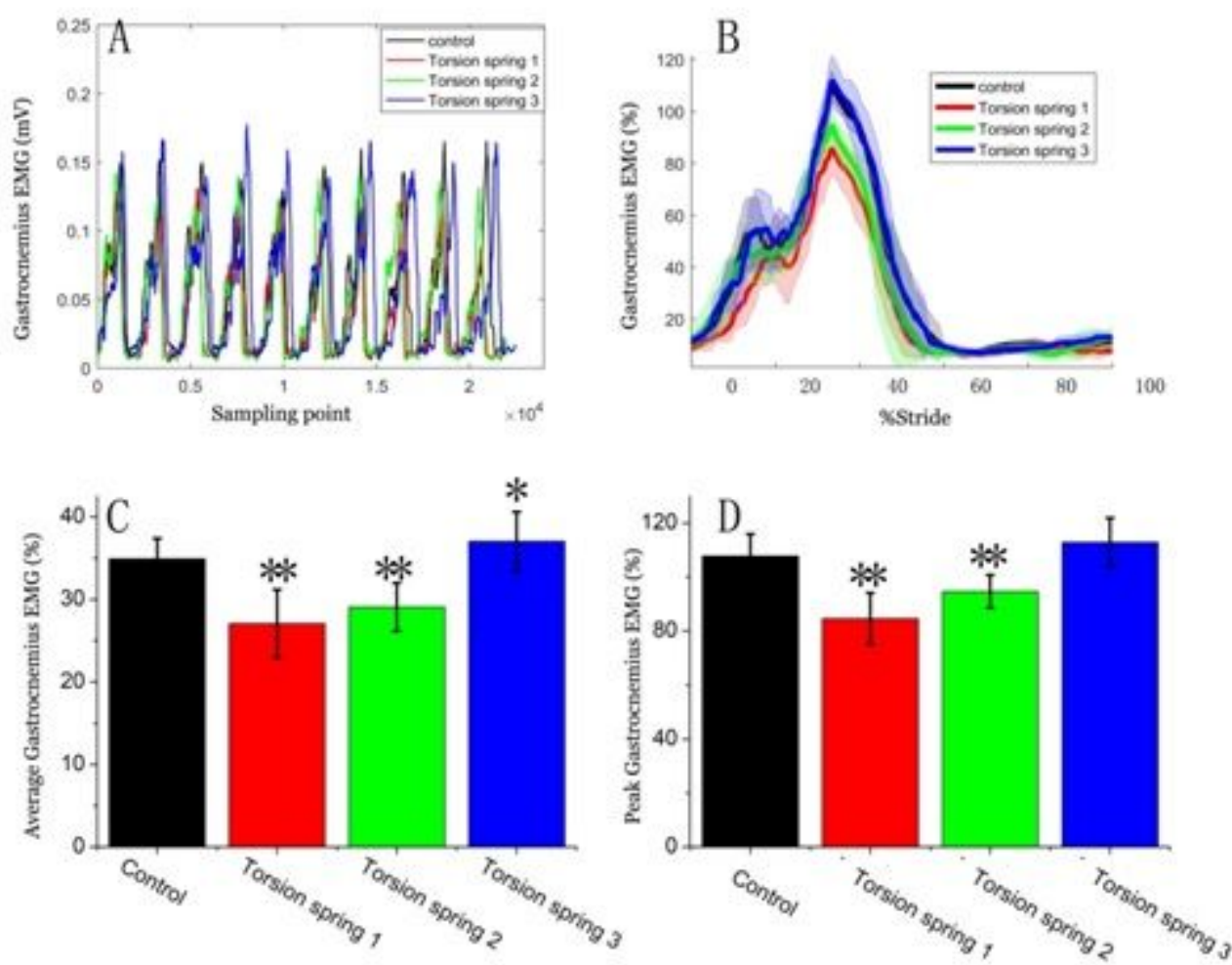

\section{Figure 6}

The gastrocnemius muscle EMG results of a typical subject. (A) RMS of the 10 strides. (B) Normalized RMS between conditions,colored lines indicate the population average of different conditions and Shaded areas indicate standard error. (C) iEMG of different conditions. (D) Peak EMG of different conditions. T test, $\mathrm{n}=10,{ }^{*} \mathrm{p} \leq 0.05$. ${ }^{*} \mathrm{p} \leq 0.01$. 

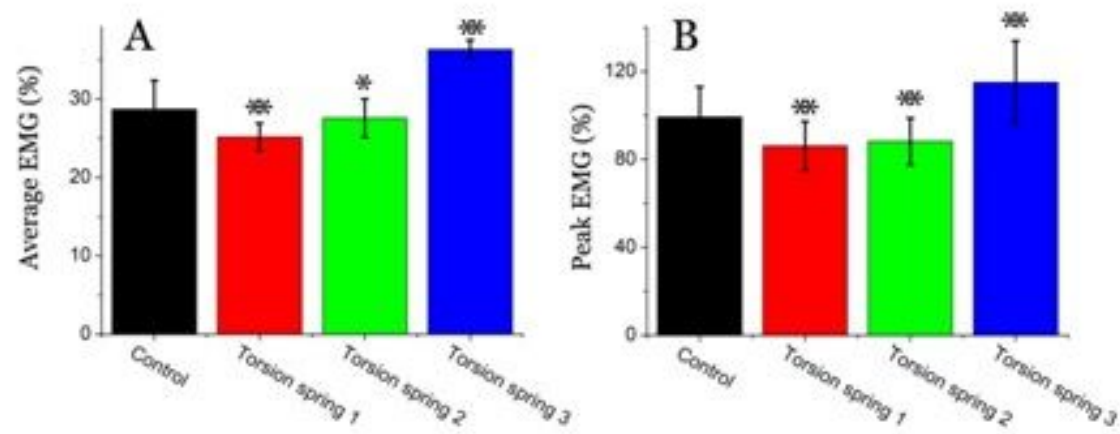

Figure 7

The gastrocnemius muscle EMG results of all subjects: $A$ is the iEMG result, $B$ is the Peak EMG result. T test, $n=80,{ }^{*} \mathrm{p} \leq 0.05 .{ }^{* *} \mathrm{p} \leq 0.01$. 